\title{
TRANSFERÊNCIA DE TECNOLOGIA PARA O CAMPO: as inovações em implementos agrícolas na região do Mato Grosso
}

Marcos Scarabelot BOZA ${ }^{1}$

Adriana Carvalho Pinto VIEIRA ${ }^{2}$

Julio Cesar ZILLI ${ }^{3}$

\author{
${ }^{1}$ Universidade do Extremo Sul Catarinense. marcos_boza@hotmail.com \\ ${ }^{2}$ Universidade do Extremo Sul Catarinense.dricpvieira@gmail.com \\ ${ }^{3}$ Universidade do Extremo Sul Catarinense. zilli42@ @otmail.com
}

Recebido em: 29/02/2016 - Aprovado em: 28/03/2017 - Disponibilizado em: 01/07/2017

\begin{abstract}
RESUMO
A agricultura passou por diversas transformações produtivas, impulsionadas pela ciência e tecnologia, bem como pela demanda de alimentos. Este avanço fomentou diversas regiões, inclusive no Brasil, possibilitando a modernização da atividade, principalmente a partir da Revolução Verde. O setor agrícola, em termos de inovação é um mercado promissor, em decorrência do alto número de equipamentos usados, cada um com funções diferenciadas. Decorrente do contexto favorável em relação à expansão e inovação do agronegócio, em especial no cenário estudado, a pesquisa tem como objetivo descrever os benefícios da utilização do rolo-corrente apresentados pelos produtores rurais do Estado do Mato Grosso. O produto, foco do presente estudo, é fabricado por uma metalúrgica situada em Turvo - SC, uma empresa de pequeno porte. A empresa fabrica outros tipos de implementos, mas o seu foco principal é o rolo-corrente, produto com pouco tempo de existência e considerado novo no mercado. Sem registros sobre o mesmo, uma pesquisa para entender a origem e descrever s benefícios seria de grande utilidade. Diante deste cenário, foram analisadas as teorias sobre inovações e implementos, e a partir da aplicação de um questionário, foram identificados os dados sobre a utilização e satisfação do rolo-corrente pelos agricultores. O resultado da pesquisa constatou que todos os entrevistados ficaram satisfeitos com os resultados do implemento e não possuem reclamações. Com estes resultados identificam-se os principais benefícios que o agricultor procura em um implemento, como redução de custos, redução de mão de obra e eficiência no resultado final no uso do produto.
\end{abstract}

Palavras chave: Inovação, Agricultura, Implementos Agrícolas.

\begin{abstract}
Agriculture has undergone several changes in production, driven by the science and technology as well as the demand for food. This advance has promoted various regions, including Brazil, enabling the modernization of the activity, mainly from the Green Revolution. The agricultural sector in terms of innovation is a promising market, due to the high number of used equipment, each with different functions. Resulting from the favorable context in relation to the expansion and agribusiness innovation, particularly in the studied scenario, the research aims to describe the benefits of using the roller-chain submitted by farmers in the state of Mato Grosso. The product focus of this study is manufactured by a metallurgical located in Turvo - SC, a small company. The company manufactures other types of implements, but its main focus is the roll-stream product with short existence and considered new in the market. No records on it, a survey to understand the origin and describe s benefits would be useful. In this scenario, was analyzed the theories of innovations and implements, and from the application of a questionnaire, was raised data on the use and satisfaction of roller-chain by farmers. The result of the survey found that all respondents were satisfied with the results of the implement and has no complaints. With these positive results can get up the main benefits the farmer looking for an implement, such as cost reduction, hand-cutting work and efficiency in the final result in the use of the product
\end{abstract}

Key-words: Innovation, Agriculture, Agricultural Implements 
A economia e a sociedade civil atravessaram um período de grandes transformações, que modificaram de forma radical, os paradigmas e a dinâmica que caracterizou a evolução nas ultimas décadas. Para assegurar a sobrevivência do ser humano e a melhoria da qualidade de vida houve a reestruturação do capitalismo, as fontes de crescimento e poder se deslocaram para o domínio do conhecimento. Assim, a Ciência, Tecnologia e Inovação (C,T\&I) e produtos do conhecimento são atualmente as molas propulsoras do desenvolvimento da economia e da sociedade (VIEIRA; BUAINAIN, 2012). Neste mesmo sentido, a agricultura passou por diversas transformações produtivas, impulsionadas pela ciência e pela intensificação tecnológica, bem como por uma maior demanda de alimentos. Este avanço fomentou diversas regiões, inclusive no Brasil, possibilitando a modernização da atividade, principalmente a partir da Revolução Verde (LOPES; SARTI; OTERO, 2014).

Decorrente destas inovações, a produção do agronegócio brasileiro cresceu exponencialmente nas ultimas décadas, haja vista que em 1975 a colheita de grãos era de 45 milhões de toneladas, expandindo para 58 milhões em 1990 e na safra 2013/2014 atingiu 192,8 milhões de toneladas. Para a safra 2014/2015 os levantamentos já apontam um acréscimo de $8,1 \%$, do qual a produção pode chegar a 201,6 milhões de toneladas (FIESC, 2014).

De acordo com Fornazier e Vieira Filho (2012) a agropecuária brasileira é profundamente heterogênea em todos os aspectos, e o acesso à tecnologia e difusão dos novos conhecimentos e técnicas se dá de forma assimétrica entre regiões, produtores e até sistemas. Ainda, no Brasil classificam a agricultura em dois tipos: o pequeno que também é conhecido como agricultura familiar e o grande produtor. Buainain, Romeiro e Guanziroli (2003) e Guanziroli, Buainain e Sabatto (2012) entendem como pequenos produtores (agricultura familiar) aqueles que possuem uma média de área para plantio até quatro módulos fiscais segundo a Lei $\mathrm{n}^{\circ} 6.746 / 79$ e é considerado grande produtor quando sua área é superior a este número. O módulo fiscal é uma unidade relativa de área, expressa em hectares, fixada para cada município, que leva em conta: tipo de exploração predominante no município; a renda obtida com a exploração predominante; outras explorações existentes no município que, embora não predominantes, sejam expressivas em função da renda ou da área utilizada; conceito de propriedade familiar.

No entanto, ao longo das décadas houve uma mudança na configuração da agropecuária brasileira, principalmente decorrente das alterações na composição da produção, 
necessitando de forças mecânicas e novas tecnologias para os trabalhos agrários. Houve aumento da utilização de insumos mais modernos e implementos agrícolas (BOLLIGER, 2014). Estas mudanças foram utilizadas tanto pela agricultura familiar como pela patronal, pois conforme Vieira Filho e Silveira (2011) os produtores adotam a tecnologia com o intuito de aumentar a sua produtividade e, simultaneamente, reduzir o custo relativo a produção.

O setor agrícola, em termos de inovação, é um mercado promissor, em decorrência do alto numero de equipamentos usados, cada um com funções diferenciadas. Neste setor, empresas de pequeno porte são responsáveis na maioria das vezes por essas inovações, para a melhoria do homem no campo, tentando encontrar meios para não prejudicar o meio ambiente, ou pelo menos na tentativa em minimizar os danos causados por maquinas, com o intuito de diminuir ao máximo os trabalhos realizados repetitivamente. Estes implementos também são usados para transporte de maquinas até os locais desejados, para carga e descarga de insumos e de outros afazeres, preparo da terra para o plantio, espalhar insumos e fertilizantes, processo de plantio e processo de colheita.

Segundo Spat (2010), a indústria de máquinas e implementos agrícolas é bastante heterogênea, pois apresenta uma estrutura econômica e produtiva que faz parte de uma cadeia produtiva que abrange diversos e distintos setores, envolvendo relações interindustriais. O setor é composto por empresas de grande e pequeno porte, de capital estrangeiro e nacional, e que se dedicam à produção de bens que são bastante distintos.

Diante do aumento da procura, o mercado de maquinas agrícola teve uma grande evolução, com as inovações de implementos e equipamentos mecanizados, como por exemplo, tratores, plantadeiras, colheitadeiras, entre outros. E dentre estas inovações, houve a criação de implementos para auxiliar a produção agrícola, como exemplo o rolocorrente, objeto de estudo. Os responsáveis por essas inovações e adaptações, na maioria das vezes são metalúrgicas, geralmente empresas de pequeno porte, por serem ágeis no atendimento aos agricultores e beneficialos em seus processos e mais atentas às necessidades dos produtores rurais.

Decorrente do contexto favorável em relação à expansão e inovação do agronegócio, em especial no cenário estudado, esta pesquisa tem como objetivo descrever os benefícios da utilização do rolo-corrente apresentados pelos produtores rurais do estado do Mato Grosso.

O estudo está estruturado em quatro seções. A primeira é a fundamentação teórica, a qual se faz uma explanação e contextualização do conceito de inovação e a inovação dos implementos agrícola. A segunda trata dos procedimentos metodológicos utilizados para a pesquisa. A terceira faz a análise dos dados 
da pesquisa. Por fim são as considerações finais e as referencias bibliográfica.

\section{Referencial Teórico: Inovação}

As inovações em produtos têm impulsionado muitas transformações no mundo. Muitas inovações são criadas e vêm acompanhando o ritmo das mudanças. Segundo Trott (2012, p. 58): “[...] o padrão de consumo refere-se ao grau de mudança exigido em relação ao pensamento e comportamento do consumidor para que utilize um produto". Ressalta-se ainda, a diferenciação de inovação e invenção, uma vez que a invenção é a criação de algo totalmente novo ou algo que tenha novidades com relação a modelos anteriores. Para que uma invenção possa se tornar uma inovação é necessária à introdução e aceitação do mercado (BARBIERI; ÁLVARES 2003).

Sendo assim, produtos que tem menor impacto em relação à poluição e a degradação da natureza, tem conquistado um maior nicho de mercado. Foi a partir desta perspectiva que o "rolo-corrente" foi idealizado e desenvolvido para que possa propiciar uma redução na quantidade de poluentes ocasionados pelos tratores e maquinas durante as operações nas lavouras.

Para Tidd, Bessant e Pavitt (2008) a inovação é uma questão que requer conhecimento, que cria novas possibilidades por meio da combinação de diferentes conjuntos de conhecimento. Tal conhecimento pode existir a partir da experiência ou resultar de um processo de busca por tecnologias, mercados, ações de concorrência, etc.

$\mathrm{Na}$ indústria de máquinas e implementos agrícola predomina o padrão de desenvolvimento tecnológico do tipo não radical e cumulativo, o que predomina é a inovação incremental (CASTRO; FONSECA, 1991).

\section{Contextualizando a inovação}

Conceituar inovação é complexo, considerando a inovação como um fator essencial para a competitividade, tendo sido fundamentada por Schumpeter, relacionando inovação ao ganho econômico. Segundo o Manual de Oslo (OECD, 1997) a implementação de um produto, bem ou serviço, seja novo ou significativamente melhorado, ou um processo, ou um novo método de marketing, ou um novo método organizacional nas práticas de negócios na organização do local de trabalho ou nas relações externas é o que se chama de "inovação". A Organização para a Cooperação e Desenvolvimento Econômico (OECD) utiliza um termo mais detalhado, inovação tecnológica de produto e processo, abreviado por 'Inovação TPP' (Technological Product and Process Innovation), que ajuda a delimitar bem o conceito de inovação.

Para o presente trabalho, a investigação está centrada na inovação de produto, ou seja, de um implemento agrícola, o qual segundo 
Tidd, Bessant e Pavitt (2008, p. 30) exemplificam:

[...] um novo modelo de carro, um novo pacote de seguros contra acidentes para bebes recém-nascidos e um novo sistema de entretenimento domestico seriam exemplos de inovação de produto. Já as mudanças nos métodos de fabricação ou nos equipamentos utilizados para produzir o carro ou o sistema de entretenimento domesticam, ou mesmo nos procedimentos administrativos, no caso do seguro, seriam exemplos de inovação de processo.

Outro ponto em que os autores focam é no grau de novidade, “[...] atualizar o modelo de um carro não é o mesmo que aparecer com um conceito de carro totalmente novo, que possua motor elétrico e seja feito de uma nova composição de matérias diferentes de aço e vidro" (TIDD; BESSANT; PAVITT, 2008, p. $30)$.

Segundo Freeman (1987) a inovação incremental ocorre com maior ou menor intensidade constantemente em qualquer indústria ou atividade de serviço, dependendo do produto. Embora esse tipo de inovação incremental possa surgir como resultado de desenvolvimento e programas organizados de pesquisa é frequente a inovação incremental ocorrer como resultado de invenção ou melhorias sugeridas por profissionais envolvidos diretamente no processo de produção ou com propostas e opiniões de usuários do produto.

Portanto, a inovação é um processo-chave na criação de produtos, processos e serviços. Ela cria um diferencial para as empresas, que as permite crescer, aumentar tanto sua produtividade quanto a qualidade de seus produtos (CASTRO, 2004).

\section{Inovação de implementos agrícolas}

A criação de implementos agrícolas surgiu a partir da necessidade. O homem deixou de ser nômade, ou seja, de ficar mudando de lugar constantemente para virar sedentário e se fixar em apenas um lugar, pois percebeu que ao plantar uma semente ela dava origem a uma planta, assim ele poderia plantar seu próprio alimento e não mais sair à procura como antes. Mas decorrente deste fato, o homem percebeu que poderia aumentar a sua própria produção. A partir disto, desenvolveu ferramentas que ajudavam o seu trabalho e com o passar dos tempos as ferramentas foram evoluindo até chegar aos implementos agrícolas (BALASTREIRE, 1990).

No final do século XVIII, a agricultura era trabalhada com instrumentos rudimentares, feitos com ferro e madeira, tudo artesanalmente. Porém, com a Revolução Industrial, o crescimento da população urbana e uma maior necessidade por alimentos, fez com que a evolução tecnológica se tornasse importante para aumentar a produtividade e assim atender a demanda crescente (FONSECA, 1990). Os EUA foi o principal país a ter desenvolvimento tecnológico na fabricação de equipamentos agrícolas, pois, no período de 1780 a 1900, a área cultivada era de 160 milhões de hectares (FONSECA, 1990). 
Neste sentido, pode ser percebido que a dinâmica tecnológica do setor de maquinas e implementos agrícolas depende na maioria das vezes de inovações externas a própria indústria. E são consideradas indústrias montadoras do setor metal mecânico, com ideias vindas diretamente do campo (DAHAB, 1993).

D acordo com Castro (2004), o agronegócio brasileiro é o setor que mais cresceu nas últimas décadas e a economia do país está praticamente sustentada a partir dele, e neste cenário, a inovação tecnológica tem uma participação muito importante. Aponta ainda o autor que, “[...] a indústria de máquinas e implementos para a agropecuária concentra boa parte das inovações tecnológicas do setor e o crescimento do agronegócio brasileiro é a mola propulsora deste segmento cada vez mais competitivo" (CASTRO, 2004, p. 3).

Portanto, os implementos agrícolas são muito importantes no desenvolvimento do trabalho diário dos agricultores, segundo Smith (2006) as inovações neste setor surgiram para facilitar os trabalhos e ajudar os agricultores a chegar aos seus objetivos finais, já que um agricultor exerce varias funções no seu dia-adia, diferentemente de pessoas que trabalham em indústrias e que exercem apenas uma função. Com a redução de tempo nos trabalhos os agricultores podem exercer outras funções com mais tempo disponível. Neste sentido, a criação de maquinas e dos implementos agrícolas protagonizou ganhos na produtividade e no trabalho agrícola, fazendo com que as técnicas de produção mudem de trajetória e, consequentemente, houve maior oferta de produtos agrícolas.

\section{Procedimentos metodológicos}

Para a classificação desta pesquisa, adota-se como referência a categorização apresentada por Vergara (2009), que classifica em relação a dois aspectos: quanto aos fins e quanto aos meios de investigação. Quanto aos fins, a pesquisa classifica-se como descritiva que tem como finalidade a descrição das características de determinada população ou fenômeno, ou então estudar as características de determinado grupo (GIL, 2002).

Quanto aos meios de investigação, apresentase como: bibliográfica e pesquisa de campo. A pesquisa bibliográfica é constituída em uma investigação em estudos científicos anteriores realizados por outros pesquisadores e que auxilia o aprofundarmos no assunto estudado, com base cientifica, conhecendo melhor o fenômeno com informações de procedência (LAKATOS; MARCONI, 1995). A pesquisa de campo, que segundo Vergara (2009) é a investigação realizada no local aonde ocorre o fenômeno, desta maneira, utilizando entrevista, aplicação de questionário ou observação.

A população alvo foi composta por agricultores do Estado do Mato Grosso que adquiriram o Rolo-corrente no período de 2008 a 2013, e que utilizam o implemento para as plantações de milheto e outras 
coberturas. Foram encaminhados os questionários para 200 clientes e houve retorno de 44 respondentes. A pesquisa foi dividida em dois blocos, o primeiro com informações sobre o proprietário e a propriedade, e no segundo bloco com parte perguntas de satisfação com relação ao "rolocorrente".

\section{Apresentação e discussão dos}

\section{resultados}

A análise dos dados foi divida em dois momentos. A primeira se refere às informações sobre as características da propriedade e do proprietário, e a segunda parte, foram analisadas as respostas com relação ao "rolo-corrente" para obter os possíveis benefícios ou restrições sobre a utilização e funcionamento do mesmo.

$\mathrm{O}$ rolo corrente é um equipamento em forma de corrente com elos, cilindros, distorcedores, garras (facas) e rolamentos que, acoplados aos engates traseiros dos tratores, trabalham paralelamente

(METALURGICA

SCARABELOT, 2015).

O implemento foi criado a partir das correntes de desmatamento (correntes lisas) utilizadas nas lavouras para corrigir as imperfeições do solo pelos produtores do Estado do Mato Grosso. A partir deste uso, a empresa, incrementando a ferramenta, adicionou facas e rolos, de acordo com a Figura 1.
Figura 1- Utilização rolo corrente

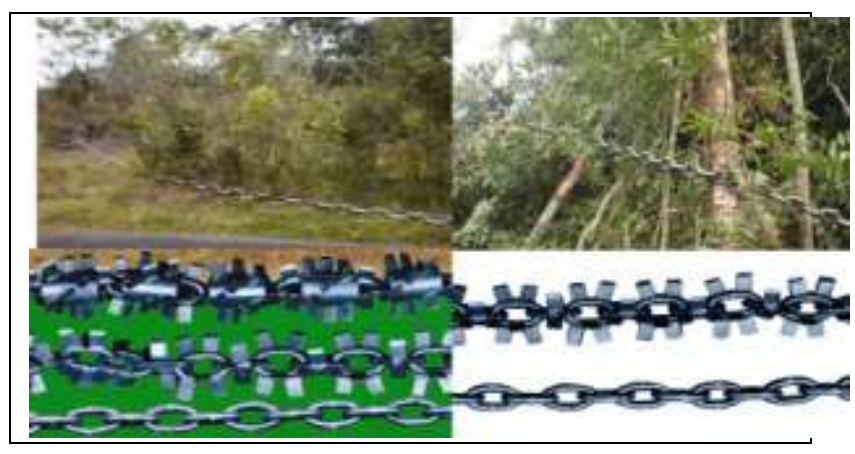

Fonte: Metalúrgica Scarabelot (2015)

O Quadro 1 apresenta uma síntese das respostas da primeira parte do questionário, com perguntas relacionadas ao contexto socioeconômico do proprietário e analise da propriedade.

Quadro 1 - Panorama socioeconômico do entrevistado

\begin{tabular}{|c|c|c|}
\hline Sexo & Masculino & $(98 \%)$ \\
\hline Área Plantada & $5.000-10.000$ ha & $(55 \%)$ \\
\hline Quantidade de tratores & 4 tratores & $(59 \%)$ \\
\hline Potencia dos tratores & $200 \mathrm{cv}$ & $(77 \%)$ \\
\hline Plantação & Milho & $(61 \%)$ \\
\hline Metragem adquirida & 80 a $90 \mathrm{mts}$ & $(45 \%)$ \\
\hline
\end{tabular}

A primeira parte do questionário foi realizada para entender a necessidade pela qual o agricultor buscou adquirir o implemento agrícola. A quantidade de área plantada, na maioria $(55 \%)$ é de 5.000 a 10.000 ha, tendo em vista que o estado do Mato Grosso é uma região com grandes quantidades de área. Com relação à quantidade de tratores, o rolocorrente utiliza dois tratores para o uso. Neste sentido, pode ser constatado que a maioria dos entrevistados têm mais de dois tratores na propriedade. 
O Mato Grosso tem uma grande variação na produção de grãos e outros produtos cultivados e o estado é considerado de fronteira agrícola. Possui $30 \%$ da área agrícola brasileira (2013) com cerca de 2,8 milhões de hectares. As áreas estão localizadas principalmente em regiões marginais e/ou frágeis, as quais requerem técnicas conservadoras, mas ao mesmo tempo sistema de produção com tecnologia. Destaca-se na produção de soja, milho, algodão e carnes, em sistemas modernos e intensivos de capital (VIEIRA JUNIOR; FIGUEIREDO; REIS, 2014).

\section{O Erro! Fonte de referência não} encontrada. apresenta os resultados da segunda parte da pesquisa, as informações sobre o implemento, negociações e trabalhos realizados.

Quadro 2 - Nível de satisfação dos produtores

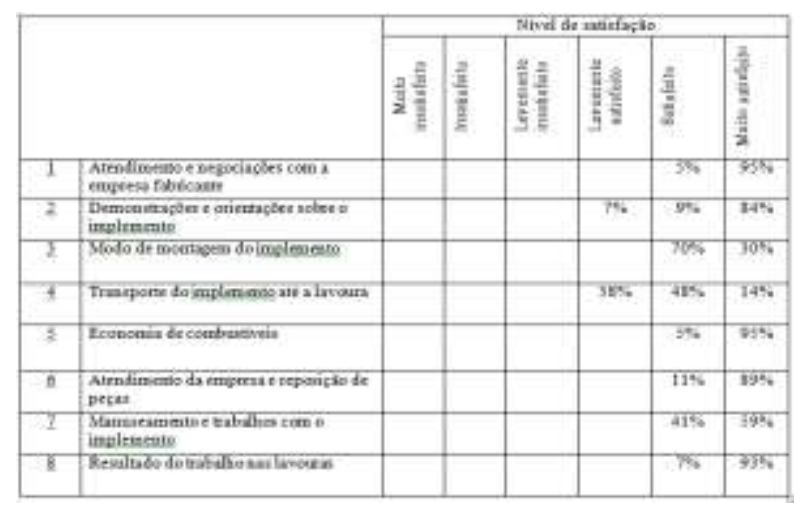

Fonte: elaboração dos autores a partir dados da pesquisa

$\mathrm{Na}$ segunda etapa, são apresentados os resultados obtidos com os questionamentos sobre a satisfação dos clientes que adquiriram o rolo-corrente. Com relação ao atendimento e negociações, os entrevistados demonstram grande satisfação com uma porcentagem de
95\%, podendo ser inferido que os produtores estão muito satisfeitos e $5 \%$ dos entrevistados apontaram que estão satisfeitos.

$\mathrm{Na}$ questão sobre demonstrações e orientações, $84 \%$ estão muito satisfeitos, $9 \%$ satisfeitos e 7\% levemente satisfeito. Esta é uma questão importante, pois é a partir das demonstrações e orientações que ser realiza a prática do produto para e com os clientes. $\mathrm{Na}$ maioria das vezes as demonstrações são realizadas em condições boas climáticas, para que o trabalho possa ser realizado perfeitamente e apresentar os resultados.

No terceiro item é apresentado dados de satisfação com relação a montagem do rolocorrente. A montagem é simples, basta direcionar as facas para o lado certo, colocar cada sessão em seu lugar e acoplar as duas pontas em dois tratores que serão puxadas.

Em relação ao transporte, os resultados que se referem ao levemente satisfeito aumentaram, pois, ao ser descarregado na propriedade, o produtor deve ter algum tipo de locomoção para transportar o rolo-corrente até a lavoura e isso pode ser feito de duas formas: com a ajuda de um caminhão ou uma carreta para ser puxada pelo próprio trator.

Em relação à economia de combustível, o implemento tem grande destaque sobre outros e por alguns motivos, dentre eles o menor número de tratores utilizados, pois no caso do rolo-corrente é utilizado apenas dois.

No sexto questionamento, $89 \%$ dos clientes estão muito satisfeitos e $11 \%$ estão satisfeitos com relação ao atendimento e reposição de 
peças. Um fator importante para esse alto nível de satisfação é sua estrutura, ele é basicamente uma corrente com tubos e facas soldados em sua composição. Um ponto fraco destacado são as facas que podem quebrar, às vezes por solda malfeita ou em razão de muitos atritos com pedras e outras formas de irregularidade no terreno. Esta ação não interfere no rendimento do implemento, mas com falta de muitas esporas e o não reposicionamento no futuro pode vir a baixar. $\mathrm{Na}$ questão do manuseio e trabalhos, a satisfação teve um grau elevado novamente. O rolo-corrente é um implemento simples em termos de uso, por ser grande e pesado pode haver algum tipo de perda de tempo.

$\mathrm{Na}$ ultima questão, a uma das mais importantes $93 \%$ dos resultados foram muito satisfeitos e $7 \%$ de satisfeitos. Por ser a ultima questão na parte de nível de satisfação dos clientes, a pergunta teve a intenção de entender o trabalho final do implemento. $\mathrm{O}$ rolo-corrente pode ser usado em vários tipos de plantação e faz o trabalho de outras maquinas, pelo nível de satisfação percebe se que ele tem bom rendimento nas lavouras.

\section{Considerações Finais}

Nos dias atuais a função da inovação vai além de ser um instrumento de competitividade. Ela aprende com os clientes e transformam o conhecimento em ações, com maior agilidade. Assim, as empresas devem ficar atentas às necessidades dos clientes, dos quais indicam possibilidades da empresa inovar.

Anteriormente, os implementos agrícolas e outras ferramentas utilizadas nos serviços de lavoura eram rudimentares. Os agricultores utilizavam basicamente o ferro e madeira para realizar os serviços. Destaca-se ainda outra dificuldade enfrentada era a falta de equipamentos apropriados e a utilização da mão de obra braçal para realizar o trabalho, tanto os leves como os pesados. Em algumas ocasiões havia a utilização de animais para a realização dos serviços na agricultura.

Com decorrer do tempo às inovações agrícolas foram surgindo, a criação de maquinas e implementos agrícolas foram substituindo o uso de mão de obra e de pessoas e animais, e substituindo implementos antigos e de baixo rendimento. A exemplo das maquinas movidas a combustíveis como, tratores e maquinas de colheita e, como exemplo de implementos utilizados para preparo de terras para plantio. Conforme aponta Terra (2012) citando Christensen a partir da evolução das inovações no campo, foi importante entender qual são as tarefas que os produtores pretendem realizar, olhando principalmente para as suas necessidades. Ainda, é necessário compreender as grandes tendências e descontinuidades econômicas, demográficas, tecnológicas e regulatórias da sociedade de uma maneira geral. E quanto à tecnologia, quais rupturas tecnológicas podem geram 
maior potencial de rápidas evoluções tecnológica.

O produto, foco do presente estudo, é fabricado por uma metalúrgica situada em Turvo-SC, considerada uma empresa de pequeno porte. A empresa fabrica outros tipos de implementos, mas o seu foco principal é o rolo-corrente, produto com pouco tempo de existência e considerado novo no mercado. Sem registros sobre o mesmo, uma pesquisa para entender a origem e descrever $\mathrm{s}$ benefícios seria de grande utilidade. Diante deste cenário, foi levantado as teorias sobre inovações e implementos, e a partir da aplicação de um questionário, foi levantado os dados sobre a utilização e satisfação do rolocorrente pelos agricultores.

$\mathrm{O}$ resultado da pesquisa constatou que todos os entrevistados ficaram satisfeitos com os resultados do implemento e não tem reclamações. Com estes resultados positivos pode se levantar os principais benefícios que o agricultor procura em um implemento, como redução de custos, redução de mão de obra e eficiência no resultado final no uso do produto.

\section{Referências Bibliográficas}

\section{BALASTREIRE, L. A. Maquinas Agrícola.} São Paulo: Editora Manole, 1990.

BARBIERI, J. C. ÁLVARES, A. C. T. Inovações nas organizações empresariais. In: BARBIERI, José Carlos. Organizações inovadoras: estudos e casos brasileiros. Rio de Janeiro: Ed. FGV, 2003. p. 41-63.
BOLLIGER, F. Brasil agropecuário: duas fotografias de um tempo que passou. Org. Antonio Marcio Buainain, Eliseu Alves, José Maria da Silveira, Zander Navarro. O mundo rural no Brasil do século XXI. Brasília: Embrapa, 2014, p. 1051-1080.

BUAINAIN, A. M.; ROMEIRO, A.; GUANZIROLI, C. Agricultura familiar e o novo mundo rural. Sociologias, Porto Alegre, n. 10, 2003. Disponível em $<$ http://www.scielo.br/scielo.php?script=sci_a rttext\&pid=S1517$45222003000200011 \& \operatorname{lng}=$ pt\&nrm=iso $>$. acessos em 22 abr. 2014.

CASTRO, B. H. R. Estratégias de inovação: um estudo na indústria de máquinas e implementos agrícolas no Brasil. Dissertação (Mestrado em Administração). Rio de Janeiro: UFRJ/COPPEAD, 2004.

CASTRO, A. C.; FONSECA, M. G.D. Inovações e concorrência em máquinas agrícolas e sementes: observações para uma nova agenda de pesquisas. Cadernos de Ciência \& Tecnologia. Brasília, v.8, n.1/3, p.48-67, 1991.

DAHAB, S. Competitividade da Indústria de Máquinas Agrícolas - Nota Técnica Setorial do Complexo Metal-Mecânico. In: Estudo da competitividade da indústria brasileira / coordenação geral Luciano G. Coutinho e João Carlos Ferraz. Campinas, SP: Papirus, 1993.

FIESC. Federação das Indústrias do Estado de São Paulo. Informativos Deagro. Dez 2014. Disponível em:

$<$ http://www.fiesp.com.br/indices-pesquisase-publicacoes/safra-de-graos-brasil/> . Acesso em: 1 maio 2015.

FONSECA, M. D. G. D. Concorrência e progresso técnico na indústria de máquinas para agricultura: um estudo sobre trajetórias tecnológicas. 1990.

FORNAZIER, A.; VIEIRA FILHO, J.E.R. Heterogeneidade estrutural no setor agropecuário brasileiro: evidencias a partir do Censo Agropecuário de 2006. Texto para 
Discussão. $\mathbf{n}^{\circ}$ 1708. Rio de Janeiro: IPEA, 2012. Disponível em:

http://agencia.ipea.gov.br/images/stories/PDF s/TDs/td_1708.pdf. Acesso em: 05 mar 2014.

FILHO, J. M. Difusão de inovações: críticas e alternativas ao modelo dominante. Caderno de Ciencia \& Tecnologia, V. 6, n.1. jan./abr. Brasília, 1989.

FREEMAN, C. Technology policy and economic performace: lessons from Japan, London. Frances Pinter. 1987.

GUANZIROLI, C. E.; BUAINAIN, A. M; SABBATO, A. Dez anos de evolução da agricultura familiar no Brasil (1996-2006). Revista de Economia e Sociologia Rural. vol. 50, n. 2. Brasília: SOBER, abril/jun, 2012.

LAKATOS, E.M.; MARCONI, M.A. Metodologia cientifica. São Paulo: Atlas, 1995.

LOPES, M.A.; SARTI, F.; OTERO, M. Apresentação livro o Mundo Rural no Brasil no Século XXI. Org. Antonio Marcio Buainain, Eliseu Alves, José Maria da Silveira, Zander Navarro. O mundo rural no Brasil do século XXI. Brasília: Embrapa, 2014.p.1-2.

\section{METALURGICA SCARABELOT. Rolo} corrente scarabelot. Disponível em: $<$ http://www.metalurgicascarabelot.com.br/rol o-corrente/>. Acesso em: 1 maio 2015.

OECD. The measurement of scientific and technological activities proposed guidelines for collecting and interpreting technological innovation data: Oslo Manual. OECD, 1997. Disponível em:

<http://www1.oecd.org/publications/ebook/9297031E.pdf.>. Acesso em: 10 mar. 2015.

ROGERS, E.M. Diffusion of innovations. $3^{\mathrm{a}}$ ed. New York: Free Press, 1995.

SMITH, A. A Riqueza das Nações. Curitiba: Juruá, 2006.
SPAT, M.D. Evolução da inovação tecnológica na indústria de máquinas e implementos agrícolas. In: Anais IV

Encontro de Economia Catarinense. Criciúma: UNESC, 2010. Disponível em: <http://www.apec.unesc.net/IV_EEC/sessoes _tematicas/Economia\%20industrial,\%20ci\%E Ancia,\%20tecnologia\%20e\%20inova\%E7\%E 3o/Evolu\%E7\%E3o\%20da\%20inova\%E7\%E 3o\%20tecnol\%F3gica\%20na\%20ind\%FAstria $\% 20 \mathrm{de} \% 20 \mathrm{~m} \%$ E1quinas $\% 20 \mathrm{e} \% 20 \mathrm{implement}$ os\%20agr\%EDcolas.pdf.> Acesso em: 1 maio 2015.

TIDD, J.; BESSANT, J.; PAVITT, K. Gestão da inovação. Rio de Janeiro: Bookman, 2008.

VERGARA, S. C. Métodos de coleta de dados no campo. Atlas, 2009.

TERRA, J.C. 10 dimensões da gestão da inovação: uma abordagem para a transformação organizacional. Rio de Janeiro: Elsevier, 2012.

TROTT, P. Gestão da inovação e desenvolvimento de novos produtos. $4^{\mathrm{a}}$. Edição. Porto Alegre: Bookman 2012

VIEIRA, A.C.P.; BUAINAIN, A.M. Aplicação da propriedade intelectual no agronegócio. In: Propriedade intelectual na agricultura. Charlene Maria Coradini de Ávila Plaza, Patrícia Aurélia Del Nero, Maria Cristina Vidotte Blanco Tarrega, Nivaldo dos Santos (coord.). Belo Horizonte: Fórum, 2012.p. 21-50.

VIEIRA F., J.E.R.; SILVEIRA, J.M.F.J. Modelo evolucionário de aprendizado agrícola. Revista Brasileira de Inovação. v.10, n.2, 2011, p.265-300.

VIEIRA JUNIOR, P.A.V.; FIGUEIREDO, E.V.C.; REIS, J.C. Alcance e limites da agricultura para o desenvolvimento regional. Org. Antonio Marcio Buainain, Eliseu Alves, José Maria da Silveira, Zander Navarro. O mundo rural no Brasil do século XXI. Brasília: Embrapa, 2014.p.127-1156. 